УДК 621.396 .67

\title{
ПЕЧАТНАЯ ПЛАНАРНАЯ НЕСИММЕТРИЧНАЯ ВИБРАТОРНАЯ АНТЕННА ДЛЯ СВЕРХШИРОКОПОЛОСНОЙ СВЯЗИ
}

\author{
Н. А. МУРУГАН ${ }^{1}$, Р. БАЛАСУБРАМАНИАН ${ }^{1}$, Х. Р. ПАТНАМ ${ }^{2}$ \\ ${ }^{1}$ SRM университет, \\ Индия, Ченнаи, Тамилнад \\ ${ }^{2}$ SAMEER, \\ Индия, Ченнаи, 600113
}

\begin{abstract}
Аннотация. В статье представлена сверхширокополосная (СШП) планарная печатная антенна с микрополосковым возбуждением. Конфигурация антенны содержит скошенную площадку заземления. Частично скошенная площадка заземления улучшает ширину полосы пропускания, определяемую по полному входному сопротивлению. Измеренная частотная характеристика показывает, что изготовленная антенна имеет ширину полосы пропускания, определяемую по полному входному сопротивлению, равную 7,9 ГГц в полосе 3,1-11 ГГц при КСВН < 2. Предложенная антенна имеет сверхширокополосные характеристики с всенаправленной диаграммой направленности и излучением с постоянным усилением. Сверхширокополосные параметры предложенной антенны оценивались с помощью распределения поверхностного тока. Результаты измерений подтверждают, что антенна может быть использована в широкополосной связи благодаря ее компактным размерам и соответствующим параметрам.
\end{abstract}

Ключевые слова: скошенное заземление; микрополосковая линия; печатный несимметричный вибратор; сверхширокополосный

\section{1. ВВЕДЕНИЕ}

Преимущества широкополосных систем проявляются при значительных скоростях. Множество СВЧ технологий работает в сверхширокополосном спектре (СШП), используя ультракороткие импульсы порядка наносекунд. СШП-системы являются привлекательными, поскольку федеральная комиссия связи FCC (Federal Communications Commission) [1] выделила полосу частот шириной полосы как минимум 500 МГц из 7,5 ГГц спектра в полосе 3,1-10,6 ГГц.

Главная цель СШП - возможность достижения высокоскоростной связи, стойкость к многолучевой интерференции и низкой потребляемой мощности при существующих стандартах беспроводной связи. Кроме того, использование СШП сигналов в беспроводной связи требует наличия антенны между приемопередатчиками и средой распространения.

СШП планарным вибраторным антеннам уделяется значительное внимание благодаря их привлекательным характеристикам, таким как большая ширина полосы пропускания, определяемая по полному входному сопротивлению, стабильная диаграмма направленности, простота производства и интеграции [2]. Для значительного уменьшения размеров антенны в [3-5] предложены планарные несимметричные вибраторные СШП антенны с микрополосковым возбуждением. Новые конструкции антенны также представлены в [6-16]. 


\section{БИБЛИОГРАФИЧЕСКИЙ СПИСОК}

1. Federal Communications Commission (FCC), Washington, DC, "First report and order in the matter of revision of Part 15 of the commission's rules regarding ultra-wideband transmission systems," ET-Docket 98-153, 2002.

2. Schantz, J. H. The Art and Science of Ultra Wideband Antennas. Artech House, 2005.

3. Huang, C.-Y.; Hsia, W.-C. "Planar elliptical antenna for ultra-wideband communiations," Electron. Lett., Vol. 41, No. 6, P. 296-297, Mar. 2005. DOI: 10.1049/el:20057244.

4. Liang, J.; Chiau, C. C.; Chen, X.; Parini, C. G. "Study of a printed disc monopole antenna for UWB systems," IEEE Trans. Antennas Propag., Vol. 53, No. 11, P. 3500-3504, Nov. 2005. DOI: 10.1109/TAP.2005.858598.

5. Zhang, J.-P.; Xu, Y.-S.; Wang, W.-D. "Microstrip-fed semi-elliptical dipole antennas for ultrawideband communications," IEEE Trans. Antennas Propag., Vol. 56, No. 1, P. 241-244, Jan. 2008. DOI: 10.1109/TAP. 2007.913165.

6. Ren, Y.-J.; Chang, K. "An annual ring antenna for UWB communications," IEEE Antennas Wireless Propag. Lett., Vol. 5, P. 274-276, 2006. DOI: 10.1109/LAWP. 2006.875897.

7. Chen, Z. N.; See, T. S. P.; Qing, X. "Small printed ultrawideband antenna with reduced ground plane effect," IEEE Trans. Antennas Propag., Vol. 55, No. 2, P. 383-388, Feb. 2007. DOI: 10.1109/TAP.2006.889823.

8. Li, Pengcheng; Liang, Jianxin; Chen, Xiaodong. "Study of printed elliptical/circular slot antennas for ultrawideband applications," IEEE Trans. Antennas Propag., Vol. 54, No. 6, P. 1670-1675, Jun. 2006. DOI: 10.1109/TAP.2006.875499.

9. Jung. J.; Choi, W.; Choi, J. "A small wideband microstrip-fed monopole antenna," IEEE Microwave Wireless Components Lett., Vol. 15, No. 10, P. 703-705, 2005. DOI: 10.1109/LMWC.2005.856834.

10. Lin, C.-C.; Kan, Y.-C.; Kuo, L.-C.; Chuang, H.-R. "A planar triangular monopole antenna for UWB communication," IEEE Microwave Wireless Components Lett., Vol. 15, No. 10, P. 624-626, Oct. 2005. DOI: 10.1109/LMWC.2005.856694.

11. Liang, X.-L.; Zhong, S.-S.; Wang, W.; Yao, F.-W. "Printed annular monopole antenna for ultra-wideband applications," Electron. Lett., Vol. 42, No. 2, P. 71-72, 2006. DOI: 10.1049/el:20063850.

12. Marchais, C.; LeRay, G.; Sharaiha, A. "Stripline slot antenna for UWB communications," IEEE Antennas Wireless Propag. Lett., Vol. 5, P. 319-322, 2006. DOI: 10.1109/LAWP.2006.878894.

13. Li, L.; Zhou, Z. I.; Hong, J. S.; Wang, B.-Z. "Compact ultra-wideband printed monopole antenna," Electron. Lett., Vol. 47, No. 16, P. 894-896, Aug. 2011. DOI: 10.1049/el.2011.1529.

14. Liu, J.; Zhong, S.; Esselle, K. P. "A printed elliptical monopole antenna with modified feeding structure for bandwidth enhancement," IEEE Trans. Antennas Propag., Vol. 59, No. 2, P. 667-670, 2011. DOI: 10.1109/TAP.2010.2096398.

15. Lao, J.; Jin, R.; Geng, J.; Wu, Q. "An ultra-wideband microstrip elliptical slot antenna excited by a circular patch," Microwave Opt. Technol. Lett., Vol. 50, No. 4, P. 845-846, 2008. DOI: 10.1002/mop.23240.

16. Azenui, A. C.; Yang, H. Y. D. "A printed crescent patch antenna for ultrawideband applications," IEEE Antennas Wireless Propag. Lett., Vol. 6, P. 113-116, 2007. DOI: 10.1109/LAWP.2007.891522.

17. Ray, K. P.; Ranga, Y. "Ultrawideband printed elliptical monopole antennas," IEEE Trans. Antennas Propag., Vol. 55, No. 4, P. 1189-1192, 2007. DOI: 10.1109/TAP.2007.893408. 